\title{
Parametric study for epoxy loaded PMMA microcapsules using Taguchi and ANOVA methods
}

\author{
S. Sharma, V. Choudhary* \\ Centre for Polymer Science \& Engineering, Indian Institute of Technology, Hauz Khas, 110016 New Delhi, India
}

Received 9 May 2017; accepted in revised form 26 July 2017

\begin{abstract}
In this study, we systematically investigated the effect of various process parameters, such as surfactant concentration, core-to-shell ratio taken in the initial feed, temperature and agitation speed on the core content of microcapsules. For this study epoxy loaded poly(methyl methacrylate) microcapsules were prepared by solvent evaporation method. Taguchi orthogonal array with $\mathrm{L}_{25}$ matrix was implemented to optimize the experimental parameters for such microcapsules. The signal-to-noise ratio (SNR) and analysis of variance (ANOVA) were also performed to determine the optimum parameters and significance of various parameters. Morphological characterization (optical microscopy, scanning electron microscopy and transmission electron microscopy) and particle size analysis (mean particle size and particle size distribution) was done to investigate the effect of various parameters on the prepared microcapsules. SNR analysis identified the optimum levels of various parameters as: surfactant concentration- $10 \mathrm{wt} \%$, core-to-shell ratio- $3: 1$, temperature- $40^{\circ} \mathrm{C}$ and agitation speed- $300 \mathrm{rpm}$. ANOVA analysis showed that surfactant concentration was the most significant parameter in improving the core content of such microcapsules. The findings of Taguchi method were also verified with contour plots. Maximum core content obtained under optimum conditions was $63.53 \mathrm{wt} \%$ and such microcapsules can find applications for the development of self-healing polymer composites.
\end{abstract}

Keywords: modelling and simulation, smart polymers, self-healing polymer composites, microcapsules synthesis, epoxy resin

\section{Introduction}

Nowadays there has been a considerable amount of research interest in recent years in the preparation of various healing agent filled microcapsules. These microcapsules can be used in the development of polymer self-healing composites for various applications such as aerospace, automotive, marine and building components etc. Currently, urea and melamineformaldehyde are the predominant shell wall materials in the preparation of such microcapsules [1-12]. However, the use of both shell wall materials has their own limitations and challenges. Yuan et al. [10] reported that poly(urea-formaldehyde) as a shell material was hydrophilic in nature, which could result in poor heat-resistance, poor anti-aging properties and higher content of free formaldehyde in the preparation of microcapsules. Though the use of poly(melamine-formaldehyde) resin as a shell material can increase the mechanical properties of microcapsules, but it is limited due to its high cost [13]. However, in spite of this the major challenge of these two materials is the use of formaldehyde which is not only a well-known human carcinogen, but also causes indoor air pollution. It has been reported in the literature that with the use of formaldehyde, people could suffer: irritation in nose, eyes and ear, trouble in respiration, vomiting tendency, skin irritation etc. [14-16]. Therefore, there is a need to develop new shell wall material for the preparation of such microcapsules.

The use of poly(methyl methacrylate) (PMMA) polymer as a potential shell wall material in microencapsulation process of such microcapsules has been reported in the literature [17-21]. Li et al. [17]

${ }^{*}$ Corresponding author, e-mail: veenach@hotmail.com

(C) BME-PT 
first introduced the preparation of curing agent and epoxy loaded PMMA microcapsules by solvent evaporation technique for the development of polymeric self-healing composites. Later on Li et al. [18] further investigated the effects of some processing parameters such as surfactant concentration, core-to-shell ratio, reaction temperature and agitation speed on the curing agent loaded PMMA microcapsules using a conventional one-at-a-time approach. The literature review shows that the parametric study for epoxy loaded PMMA microcapsules is limited [21]. A detailed parametric study of such microcapsules can improve the various properties of such microcapsules (i.e., yield percent, smooth and stable morphology and core content etc.) most importantly the core content (i.e. amount of healing agent within the capsules). In the selfhealing of polymer composites through microcapsules approach, core content of microcapsules plays an important role in healing of the composite material. The microcapsules containing high amount of core content can release the maximum amount of healing material which can heal the maximum area of cracked surface.

For this study four parameters such as surfactant concentration, core-to-shell ratio, temperature and agitation speed and their five different levels have been selected for experimentation. The full factorial experiments of $625\left(5^{4}\right)$ can be completed in just 25 experiments by the implementation of Taguchi orthogonal array with $\mathrm{L}_{25}$ matrix. As a result, Taguchi method is more advantageous in comparison to other traditional methods such as full factorial design and simplex etc. because it drastically reduces the number of experimental runs to a practical level. Hence, this approach significantly saved the experimental time and its related costs which could be consumed for the conduction of four parameters individually. Therefore, this methodology is more adaptable than the other methods [22-26]. In Taguchi methodology two essential tools are required such as:

(i) Taguchi orthogonal array (OA): Taguchi's OA allows considering a selected subset of combinations of multiple parameters at multiple levels. Taguchi OAs are balanced to ensure that all levels of all parameters are considered equally.

(ii) Signal-to-noise ratio (SNR) analysis: This analysis displays the relation between profitable results (signal) and deviation of measured values (noise). In addition, this ratio clarifies the optimization process i.e. higher the SNR, more optimal are the results [27, 28].

ANOVA is employed to find the optimum parameter conditions and to analyze the effect of various process parameters on the core content of such microcapsules. Thus, the aim of this article is to study the influence of various parameters on the core content of epoxy loaded PMMA microcapsules through Taguchi's parameter design approach. An optimum combination of parameter conditions obtained for surfactant concentration, core-to-shell ratio, temperature and agitation speed in response to maximizing the core content of microcapsules were evaluated and later verified experimentally. The resulting epoxy loaded PMMA microcapsules were characterized by optical microscopy (OM), scanning electron microscopy (SEM), transmission electron microscopy (TEM), mean particle size and particle size distribution (PSD). Furthermore, a comparative study between contour plots and Taguchi method was also done to verify the Taguchi's method findings.

\section{Experimental}

\subsection{Materials}

Diglycidyl ether of bisphenol-A(DGEBA) [(Araldite LY 556) having epoxide equivalent weight: 183$189 \mathrm{~g} / \mathrm{eq}$, viscosity at $25^{\circ} \mathrm{C}: 10000-12000 \mathrm{cps}$ ] used as a core material was purchased from Huntsman Advanced Materials (P) Ltd. Mumbai, India. Poly (vinyl alcohol) (PVA) $\left(M_{\mathrm{w}}\right.$ approx. 125000) and sodium laurlyl sulphate (SLS) used as stabilizer and ionic surfactant were purchased from Central Drug House (P) Ltd. India. Analytical grade dichloromethane (DCM) purchased from Fischer Scientific (P) Ltd. was used as received. Deionised (DI) water was used throughout the course of this study.

PMMA was prepared in the laboratory by bulk polymerization method using azobisisobutyronitrile (AIBN) as initiator, methyl methacrylate (MMA) as monomer and methanol as coagulant. The average molecular weight of the prepared polymer was determined by GPC analysis: $M_{\mathrm{n}}=5.38 \cdot 10^{5}$ and $M_{\mathrm{W}}=$ $8.75 \cdot 10^{5}$ daltons with polydispersity index of 1.6 .

\subsection{Preparation of PMMA microcapsules}

A series of epoxy loaded PMMA microcapsules were prepared by solvent evaporation method. All experiments were carried out in a $2 \mathrm{~L}$ beaker fitted with a mechanical stirrer. A procedure adopted for the preparation of such microcapsules is presented 


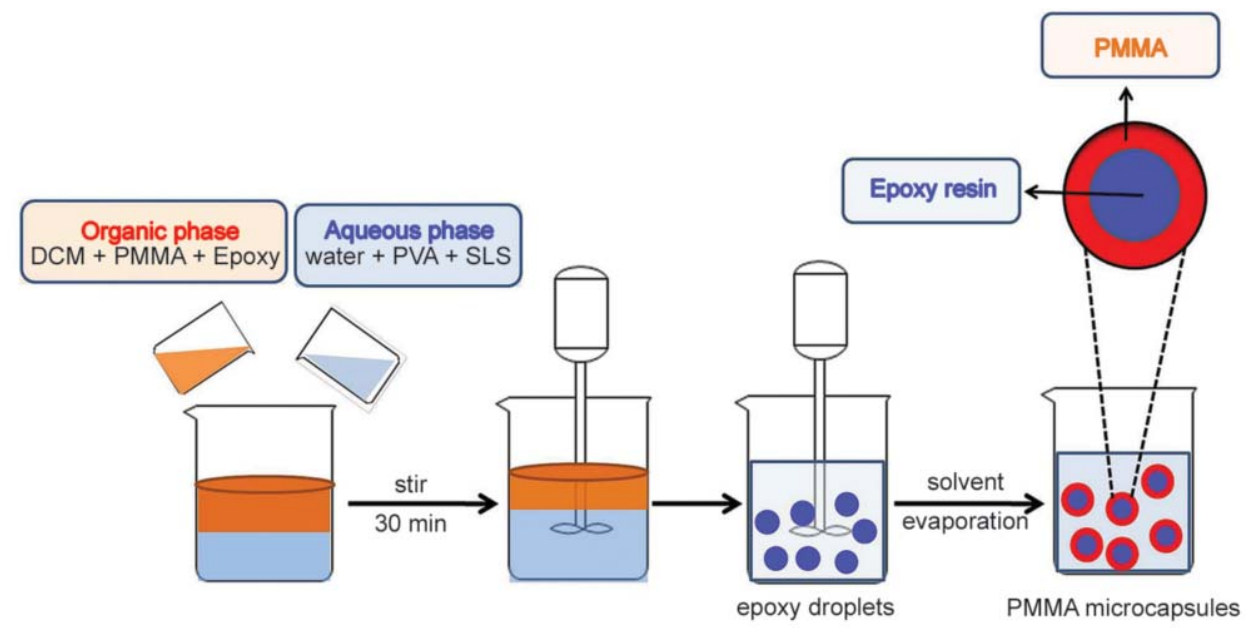

Figure 1. Schematic diagram showing the preparation of epoxy loaded microcapsules using solvent evaporation method

in Figure 1. Following steps were involved in the preparation of such microcapsules:

\section{(1) Preparation of aqueous and organic phase}

For the preparation of microcapsules two phases such as aqueous and organic phases were prepared as follows:

(i) Aqueous phase $(300 \mathrm{~mL})$ was prepared by dissolving $1 \mathrm{wt} \%$ PVA and varied amounts of SLS (as given in Table 2).

(ii) For the preparation of organic phase $1 \mathrm{~g}$ PMMA was dissolved in $30 \mathrm{~mL}$ of DCM and varied amounts of epoxy (as given in Table 2) was added.

\section{(2) Preparation of microcapsules}

For the microencapsulation process the organic phase $(30 \mathrm{~mL})$ was subsequently poured into aqueous phase $(100 \mathrm{~mL})$ under continuous agitation and the mixture was left for $30 \mathrm{~min}$ under agitation for the formation of epoxy droplets. After $30 \mathrm{~min}$, the resultant oil-in-water $(\mathrm{O} / \mathrm{W})$ emulsion was poured into remaining aqueous phase $(200 \mathrm{~mL})$ using varying agitation speed (as given in Table 2) and at varying temperature (as given in Table 2). After maintaining all experimental conditions, DCM was allowed to evaporate completely to obtain PMMA microcapsules. Finally, the prepared microcapsules were cooled down to room temperature, separated using centrifuge at $2000 \mathrm{rpm}$, washed repeatedly with methanol and DI water to remove the unencapsulated epoxy resin, remaining PVA and SLS. Then the thoroughly washed microcapsules were freeze dried for $24 \mathrm{~h}$.

\subsection{Design of experiment (DOE) by Taguchi method}

\subsubsection{Selection of parameters and their levels}

From the basic knowledge of epoxy loaded PMMA microcapsules preparation, four key parameters that could affect the core content of such microcapsules were chosen for this study i.e. (i) surfactant concentration; (ii) core-to-shell ratio; (iii) temperature and (iv) agitation speed. The selected parameters and their levels are summarized in Table 1. The ranges of levels were chosen on the basis of literature $[18,21]$ and our preliminary experiments.

\subsubsection{Selection of Taguchi's $\mathrm{OA}$}

After selection of parameters and their respective levels, selection of Taguchi's OA is the most important step. For the study of four parameter and their five levels, the $\mathrm{L}_{25} \mathrm{OA}$ can be used [29]. The twenty five combinations of the parameters are outlined in Table 2.

\subsubsection{SNR analysis}

Taguchi methodology utilizes the SNR analysis to determine the optimum conditions for process parameters. There are three types of SNR analysis criteria for optimization: smaller-the-better, larger-thebetter and nominal-the-better [30, 31]. In this study,

Table 1. Experimental parameters and their selected levels

\begin{tabular}{|c|c|c|c|c|c|c|}
\hline \multirow{2}{*}{\multicolumn{2}{|c|}{ Parameters [units] }} & \multicolumn{5}{|c|}{ Levels } \\
\hline & & 1 & 2 & 3 & 4 & 5 \\
\hline Surfactant concentr & {$[\mathrm{g}]$} & 2.5 & 5 & 7.5 & 10 & 12.5 \\
\hline Core-to-shell ratio & [in moles] & $1: 1$ & $1: 2$ & $1: 3$ & $1: 4$ & $1: 5$ \\
\hline Temperature & {$\left[{ }^{\circ} \mathrm{C}\right]$} & 25 & 40 & 55 & 70 & 85 \\
\hline Agitation speed & {$[\mathrm{rpm}]$} & 300 & 600 & 900 & 1200 & 1500 \\
\hline
\end{tabular}


Table 2. Experimental layout of the $\mathrm{L}_{25} \mathrm{OA}$, experimental results of core content and their respective SNR [dB]

\begin{tabular}{|c|c|c|c|c|c|c|c|c|c|}
\hline \multirow{2}{*}{$\begin{array}{l}\text { Run } \\
\text { [Nos.] }\end{array}$} & \multirow{2}{*}{$\begin{array}{c}\text { Surfactant } \\
\text { concentration } \\
\text { [g] }\end{array}$} & \multirow{2}{*}{$\begin{array}{l}\text { Core-to- } \\
\text { shell ratio } \\
\text { [in moles] }\end{array}$} & \multirow{2}{*}{$\begin{array}{c}\text { Temperature } \\
{\left[{ }^{\circ} \mathrm{C}\right]}\end{array}$} & \multirow{2}{*}{$\begin{array}{c}\text { Agitation } \\
\text { speed } \\
\text { [rpm] }\end{array}$} & \multicolumn{4}{|c|}{$\begin{array}{c}\text { Core content } \\
\text { [wt \%] }\end{array}$} & \multirow{2}{*}{$\begin{array}{l}\text { SNR } \\
{[d B]}\end{array}$} \\
\hline & & & & & R1 & R2 & R3 & Average & \\
\hline 1 & 2.5 & $1: 1$ & 25 & 300 & 33.0 & 35.0 & 34.0 & 34.0 & 30.63 \\
\hline 2 & 2.5 & $2: 1$ & 40 & 600 & 43.0 & 38.0 & 39.0 & 40.0 & 32.04 \\
\hline 3 & 2.5 & $3: 1$ & 55 & 900 & 37.5 & 40.0 & 39.5 & 39.0 & 31.82 \\
\hline 4 & 2.5 & $4: 1$ & 70 & 1200 & 29.0 & 27.0 & 28.0 & 28.0 & 28.94 \\
\hline 5 & 2.5 & $5: 1$ & 85 & 1500 & 8.0 & 15.0 & 10.0 & 11.0 & 20.83 \\
\hline 6 & 5.0 & $1: 1$ & 40 & 900 & 42.0 & 42.0 & 44.0 & 42.0 & 32.47 \\
\hline 7 & 5.0 & $2: 1$ & 55 & 1200 & 41.0 & 43.0 & 45.0 & 43.0 & 32.67 \\
\hline 8 & 5.0 & $3: 1$ & 70 & 1500 & 38.0 & 38.0 & 35.0 & 37.0 & 31.36 \\
\hline 9 & 5.0 & $4: 1$ & 85 & 300 & 29.0 & 32.0 & 29.0 & 30.0 & 29.54 \\
\hline 10 & 5.0 & $5: 1$ & 25 & 600 & 41.0 & 43.0 & 45.0 & 43.0 & 32.67 \\
\hline 11 & 7.5 & $1: 1$ & 55 & 1500 & 37.0 & 40.0 & 46.0 & 41.0 & 32.26 \\
\hline 12 & 7.5 & $2: 1$ & 70 & 300 & 46.0 & 54.0 & 56.0 & 52.0 & 34.32 \\
\hline 13 & 7.5 & $3: 1$ & 85 & 600 & 38.0 & 49.0 & 48.0 & 45.0 & 33.06 \\
\hline 14 & 7.5 & $4: 1$ & 25 & 900 & 45.0 & 51.0 & 51.0 & 49.0 & 33.80 \\
\hline 15 & 7.5 & $5: 1$ & 40 & 1200 & 47.0 & 45.0 & 49.0 & 47.0 & 33.44 \\
\hline 16 & 10.0 & $1: 1$ & 70 & 600 & 47.0 & 48.3 & 49.0 & 48.1 & 33.64 \\
\hline 17 & 10.0 & $2: 1$ & 85 & 900 & 44.0 & 44.0 & 41.0 & 43.0 & 32.67 \\
\hline 18 & 10.0 & $3: 1$ & 25 & 1200 & 52.0 & 55.0 & 49.0 & 52.0 & 34.32 \\
\hline 19 & 10.0 & $4: 1$ & 40 & 1500 & 51.0 & 49.0 & 50.0 & 50.0 & 33.98 \\
\hline 20 & 10.0 & $5: 1$ & 55 & 300 & 55.0 & 58.0 & 61.0 & 58.0 & 35.27 \\
\hline 21 & 12.5 & $1: 1$ & 85 & 1200 & 32.0 & 30.0 & 34.0 & 32.0 & 30.10 \\
\hline 22 & 12.5 & $2: 1$ & 25 & 1500 & 38.0 & 44.0 & 41.0 & 41.0 & 32.26 \\
\hline 23 & 12.5 & $3: 1$ & 40 & 300 & 65.0 & 62.0 & 65.0 & 64.0 & 36.12 \\
\hline 24 & 12.5 & $4: 1$ & 55 & 600 & 54.0 & 55.0 & 50.0 & 53.0 & 34.49 \\
\hline 25 & 12.5 & $5: 1$ & 70 & 900 & 40.0 & 35.0 & 45.0 & 40.0 & 32.04 \\
\hline
\end{tabular}

a high core content of microcapsules is a desirable property; hence, the statistical analysis is carried out with larger-the-better criteria. SNR for larger-thebetter is expressed according to Equation (1):

$\frac{S}{N}=-10 \cdot \log ($ mean square deviation)

$\frac{S}{N}=-10 \cdot \log \frac{1}{n\left(\sum \frac{1}{y^{2}}\right)}$

Where $y$ represents the measured value of core content obtained from a run and $n$ is the number of measurements in a trial. All statistical analyses were done using Minitab 17 software.

\subsubsection{ANOVA analysis}

In order to observe the practical (\% contribution) and statistical $(P \leq 0.05)$ significance of various parameters on core content of microcapsules, ANOVA was performed. From this analysis, variance of parameter was calculated by determining the quantities such as the degree of freedom, sum of square, variance and the percentage contribution of each parameter [31].

\subsection{Characterization of microcapsules}

The amount of epoxy resin encapsulated in the prepared PMMA microcapsules was determined by extraction method based on dissolution principle [32, 33]. For the determination of core content, an accurately weighed $\left(W_{1}\right)$ sample was crushed within a mortar-pestle. The crushed microcapsules were then mixed with methanol and stirred for $1 \mathrm{~h}$ using magnetic stirrer followed by filtration. The residue was washed several times with methanol, dried in vacuum oven for $24 \mathrm{~h}$ and weighed $\left(W_{2}\right)$ until we got a constant weight. The core content was calculated according to Equation (2):

$$
\text { Core content }=\frac{W_{1}-W_{2}}{W_{1}} \cdot 100 \%
$$

$W_{1}$ : initial weight of sample, $W_{2}$ : weight of sample after extraction with methanol.

Optical micrographs of microcapsules were recorded using OM with INSTEC HCS302 instrument at 50× magnifications. OM was used to provide information about the morphology. The morphology and shell 
wall thickness of the prepared microcapsules were also characterized by SEM using a Zeiss EVO 50 SEM machine at $20 \mathrm{kV}$. For this purpose, microcapsules were mounted on a carbon tape and coated with gold prior to imaging. For shell wall thickness analysis, known weights of microcapsules were homogeneously dispersed in the epoxy matrix. Furthermore TEM with a Jeol 1400 machine was used to confirm the core-shell morphology of prepared microcapsules.

In order to define the mean particle size and particle size distribution of the prepared microcapsules SEM images were used. For this purpose, 200 microcapsules were analyzed using ImageJ software and the mean particle size is reported.

\subsection{Contour plots}

In this study, we drew contour plots of core content to verify the results of Taguchi methodology. All contour plots were drawn using Minitab 17 software. Contour plots display a 3-dimensional relationship in two dimensions, $x$ and $y$ parameters plotted on the $x$ and $y$ scales and the response values represented by contours (colour mapping). One can think of a contour plot like a topographical map, in which the darker regions indicate the higher quality [34].

\section{Results and discussion}

\subsection{SNR analysis}

The core content was measured for the twenty five experiments and the average value of core content from three replications was calculated and summarized in Table 2. SNR were also calculated with Taguchi method for the average value of core content using larger - the-better criteria and also summarized in Table 2.

The main effects plot was drawn for each parameter at different levels by taking levels as $x$-coordinates and SNR as y-coordinates. Figure $2 \mathrm{a}$ shows that the main effects plot exhibiting the change in SNR for the average core content from which the following conclusions are drawn.

\subsubsection{Effect of surfactant concentration on the core content}

Figure 2a shows that the SNR value increases as the surfactant concentration increases. The surfactant acts as a stabilizing agent for microparticles and at optimum surfactant concentration, stable microparticles are formed which might have resulted in high core content of microcapsules and an increase in SNR value. In contrast, at very high surfactant concentration the SNR decreases. Various studies showed that very fine emulsions having smaller microcapsules are obtained with increasing surfactant concentration [35-37] which may be responsible for low core content of microcapsules and a decrease in SNR value.

\subsubsection{Effect of core-to-shell ratio on the core content}

Figure 2a shows that the SNR value increases as the core-to-shell ratio increases from 1:1 to 3:1 followed by a decrease as we further increase the core-to-shell ratio from 3:1 to $5: 1$. This has also been reported by Li et al. [18] that at a very high core-to-shell ratio, microcapsules shell wall thickness increases which in-turn resulted in the low core content of microcapsules and thus a decrease in SNR value.
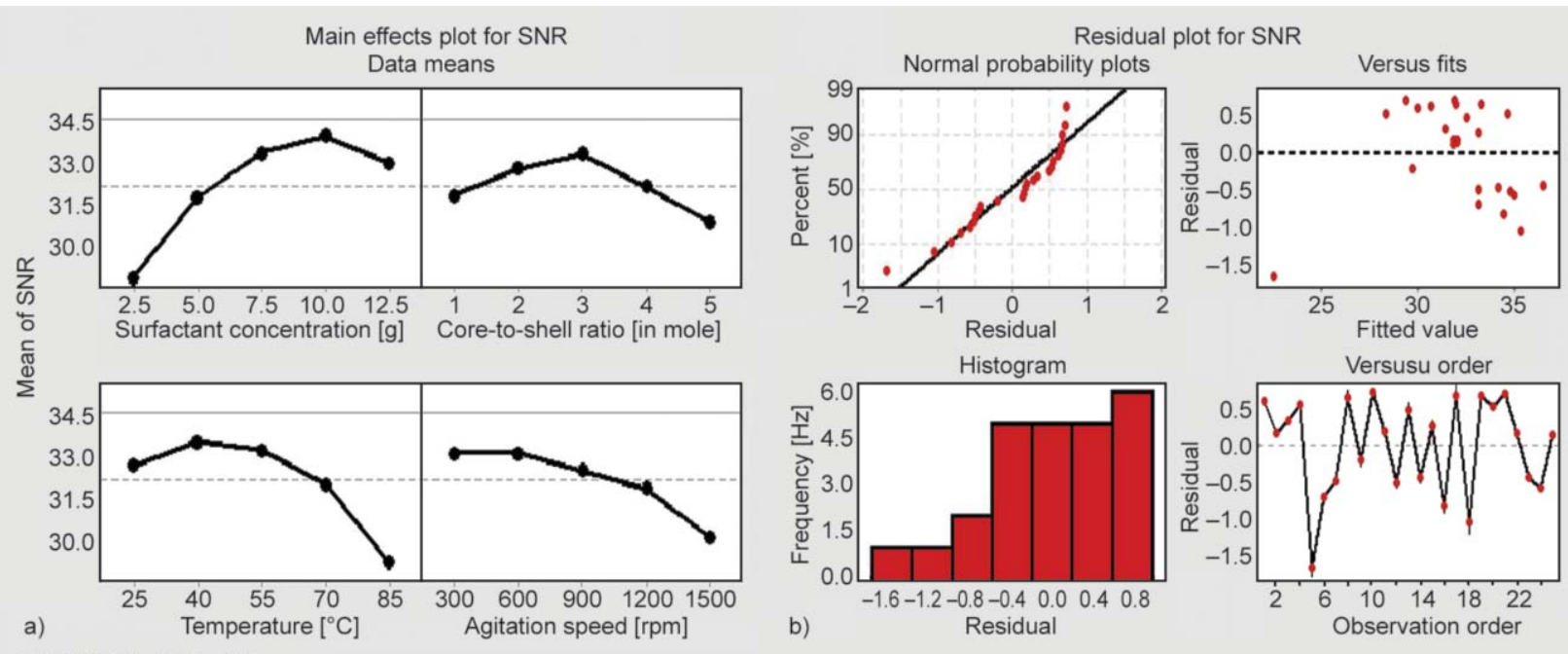

Figure 2. (a) Main effect plots and (b) residual plots used for SNR 


\subsubsection{Effect of temperature on the core content}

Figure 2a shows that the SNR value increases as the temperature increases from 25 to $40^{\circ} \mathrm{C}$ followed by a decrease as we further increase the temperature from 40 to $85^{\circ} \mathrm{C}$. In general, in solvent evaporation method the quick evaporation of solvent significantly affects the surface morphology of the microcapsules [20]. Hence, an increase in the reaction temperature leads in the formation of deep pores on the surface of microcapsules. Thus, formation of pores might have resulted in low core content of microcapsules due to easy diffusion of core material and a decrease in SNR value.

\subsubsection{Effect of agitation speed on the core content}

Figure $2 \mathrm{a}$ shows that the SNR value continuously decreases as the agitation speed increases from 300 to $1200 \mathrm{rpm}$. It was reported earlier that the capsule size and capsule shell wall thickness both decreased with increasing agitation rates [38]. Hence, an increase in the agitation speed might have resulted in the unfilled broken microcapsules, which could be a reason for low core content of microcapsules and a decrease in SNR value.

Figure $2 b$ shows the residual plots for SNR that are drawn to examine the goodness of the data. From residual plots, it is observed that the data are normally distributed in all plots.

\subsection{Optimum levels}

The response data obtained for SNR was analyzed to optimize the optimum levels as summarized in Table 3. A higher SNR value corresponds to a better performance [29]. Therefore, the optimal level of the process parameters is the level with the higher SNR value such as: level-4 for surfactant concentration, level-3 for core-to-shell ratio, level-2 for temperature and level-1 for agitation speed.
Table 3. Response table for SNR larger-the-better

\begin{tabular}{|c|c|c|c|c|}
\hline Level & \begin{tabular}{|c|} 
Surfactant \\
concentration \\
{$[g]$}
\end{tabular} & $\begin{array}{c}\text { Core-to-shell } \\
\text { ratio } \\
\text { [in moles] }\end{array}$ & $\begin{array}{c}\text { Temperature } \\
{\left[{ }^{\circ} \mathrm{C}\right]} \\
\end{array}$ & \begin{tabular}{|c|} 
Agitation \\
speed \\
[rpm]
\end{tabular} \\
\hline 1 & 28.85 & 31.82 & 32.74 & 33.18 \\
\hline 2 & 31.74 & 32.79 & 33.61 & 33.18 \\
\hline 3 & 33.38 & 33.34 & 33.30 & 32.56 \\
\hline 4 & 33.98 & 32.15 & 32.06 & 31.90 \\
\hline 5 & 33.00 & 30.85 & 29.24 & 30.14 \\
\hline Delta & 5.12 & 2.49 & 4.37 & 3.04 \\
\hline Rank & 1 & 4 & 2 & 3 \\
\hline
\end{tabular}

\subsection{ANOVA analysis}

In this study, ANOVA is used to evaluate, which process parameter significantly affects the core content property of microcapsules. Therefore, ANOVA analysis was performed on the SNR data. The results of ANOVA analysis are summarized in Table 4. From ANOVA analysis it has been illustrated, that core

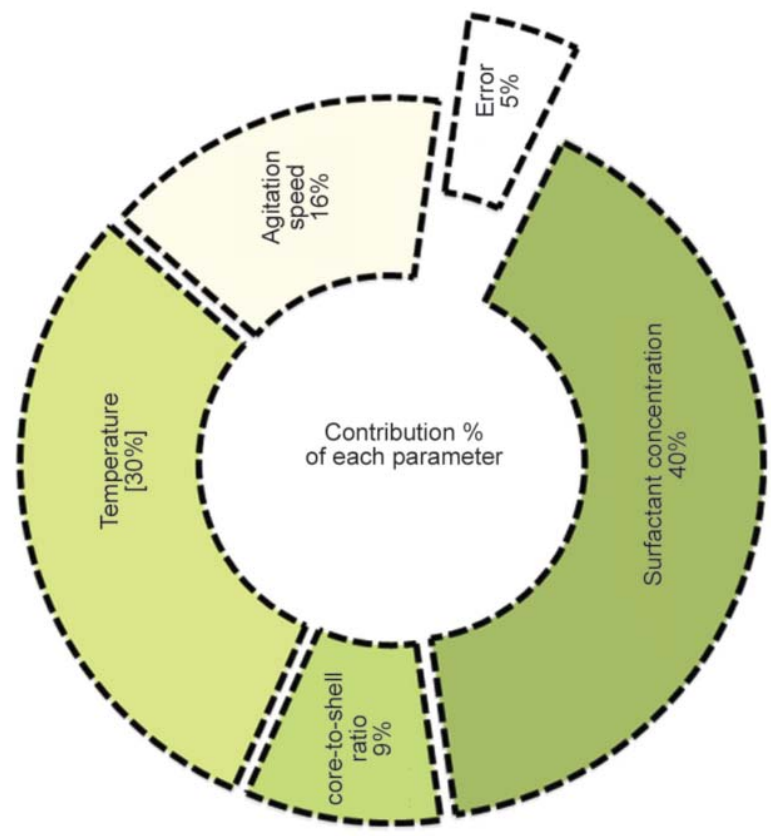

Figure 3. Contribution percentage of each parameter to the response core content

Table 4. Results from ANOVA analysis

\begin{tabular}{|l|c|r|r|r|r|r|}
\hline \multicolumn{1}{|c|}{ Parameters } & $\begin{array}{c}\text { Degree of } \\
\text { freedom }\end{array}$ & $\begin{array}{c}\text { Sum of } \\
\text { squares }\end{array}$ & Variance & $\boldsymbol{F}$-ratio & $\boldsymbol{P}$ value & Percentage contribution \\
\hline Surfactant concentration & 4 & 82.99 & 20.747 & 16.13 & 0.001 & 40 \\
\hline Core-to-shell ratio & 4 & 18.08 & 4.520 & 3.52 & $0.061^{*}$ & 9 \\
\hline Temperature & 4 & 61.29 & 15.322 & 11.92 & 0.002 & 30 \\
\hline Agitation speed & 4 & 31.98 & 7.995 & 6.22 & 0.014 & 16 \\
\hline Residual error & 8 & 10.29 & 1.286 & & & 5 \\
\hline Total & 24 & 204.62 & & & & 100 \\
\hline
\end{tabular}

*Insignificant parameter

$S=1.134 ; R-S q=95.0 \% ; R-S q_{(\text {adj })}=85 \%$ 
content of microcapsules are significantly affected by these three parameters in the following order: surfactant concentration $(40 \%)>$ temperature $(30 \%)>$ agitation speed (16\%). The contribution percentage of all parameters is presented in Figure 3. This analysis is based on the higher values of $F$ ratio and lower values of $P[29,30]$. Hence, on the basis of ANOVA analysis, surfactant concentration is considered as the most significant process parameter in controlling the core content of PMMA microcapsules. This may be attributed to the fact, that the presence of surfactant in the preparation of microcapsules may be responsible to produce a uniform protective layer around the epoxy droplets, which could be responsible for better stability of microcapsules. Thus, with the increase of surfactant concentration the core content significantly increased.
On the other hand, core-to-shell ratio was least effective parameter (contributing only $9 \%$ ) among all or one can say insignificant parameter in controlling the core content of PMMA microcapsules.

$R-S q=95.0 \%$ indicated that the ANOVA analysis was able to predict the response with high accuracy.

\subsection{Confirmatory experiments for optimal conditions}

Confirmatory experiments were performed using optimum process of parameter. The average value of three experimental results of the confirmatory experiments is summarized in Table 5. It is important to mention here that predicted mean value (as shown in Table 5) was calculated using Minitab 17 software. The experimental results correspond closely with the predicted result (error between the predicted

Table 5. Results of the confirmatory experiments

\begin{tabular}{|c|c|c|c|c|c|c|}
\hline \multicolumn{4}{|c|}{ Input parameters } & \multicolumn{3}{|c|}{$\begin{array}{c}\text { Output response core content } \\
{[w t \%]}\end{array}$} \\
\hline $\begin{array}{c}\text { Surfactant } \\
\text { concentration } \\
{[\mathrm{g}]}\end{array}$ & $\begin{array}{c}\text { Core-to-shell ratio } \\
\text { [in mole] }\end{array}$ & $\begin{array}{c}\text { Temperature } \\
{\left[{ }^{\circ} \mathrm{C}\right]}\end{array}$ & $\begin{array}{c}\text { Agitation } \\
\text { speed } \\
\text { [rpm] }\end{array}$ & Prediction & Experimental & Error \\
\hline 10 & $3: 1$ & 40 & 300 & 66.36 & 63.53 & $2.83 \%$ \\
\hline
\end{tabular}

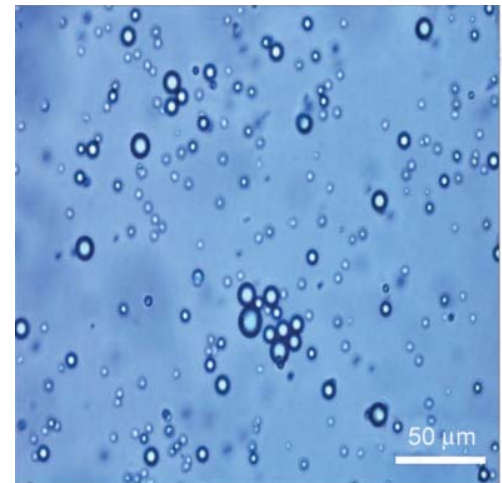

a)

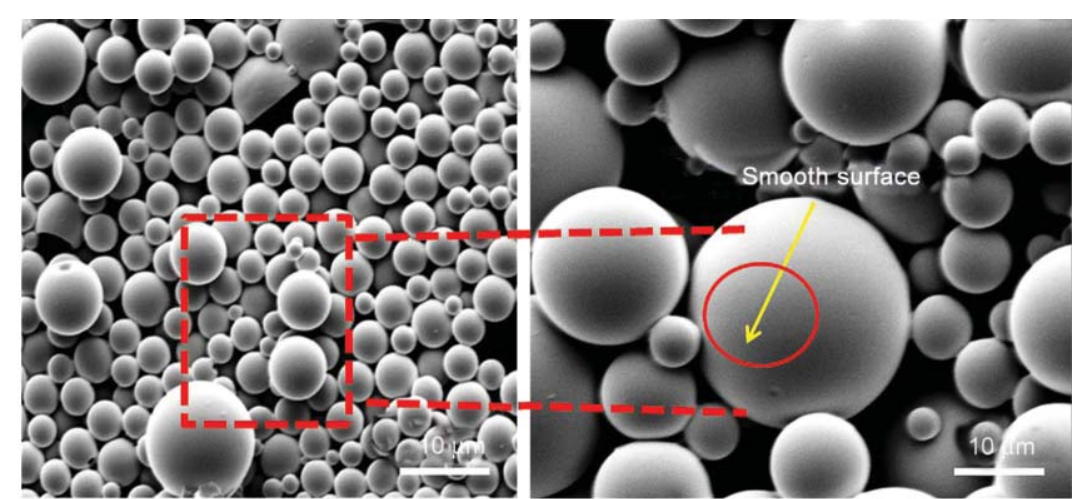

b)

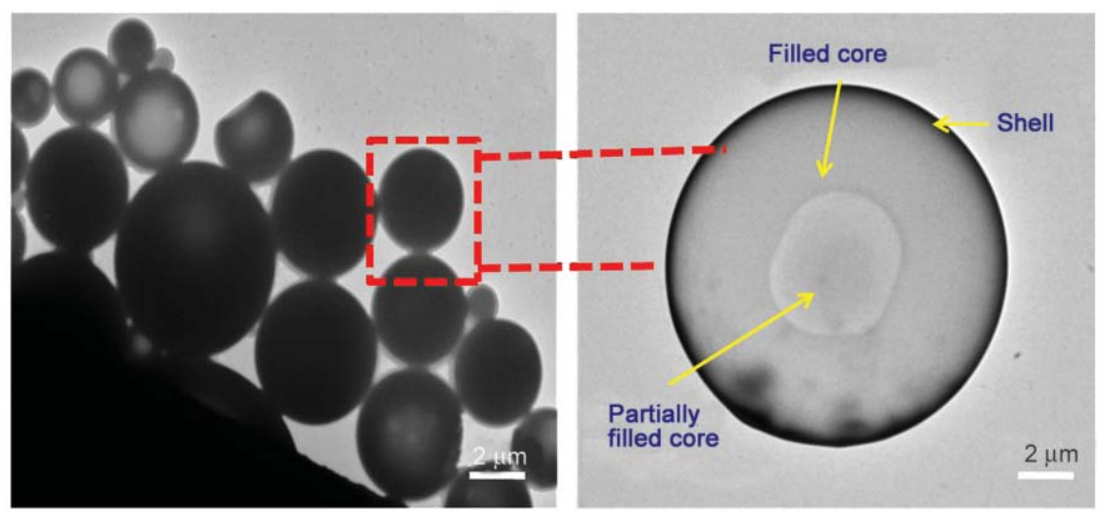

c)

Figure 4. Images of microcapsules prepared under optimum conditions (a) OM micrograph of microcapsules at 50× magnification, (b) SEM micrographs of microcapsules at 1000× and 5000× magnifications and (c) TEM micrographs of microcapsules at $500 \times$ and $2000 \times$ magnifications 
and actual result is less than $3 \%$ ). Therefore, the Taguchi method is found effective for the optimization of process parameter for the preparation of PMMA microcapsules.

\subsection{Characterizations of microcapsules}

Figure 4 shows the morphology of epoxy loaded PMMA microcapsules prepared under optimum conditions. The OM and SEM micrographs of microcapsules are presented in Figure $4 \mathrm{a}$ and $4 \mathrm{~b}$. All micrographs showed free flowing, spherical microcapsules with distinct shell wall morphology. From SEM micrographs it is confirmed, that all microcapsules have smooth surface morphology. The TEM micrographs further confirmed the presence of well defined core and shell structures as presented in Figure $4 c$. The epoxy is seen as lighter density core which is surrounded by a dense PMMA polymer shell. The morphological results in the present study indicate a much better surface morphology of PMMA microcapsules when compared with those reported by Ahangaran et al. [21].

Figure 5 shows the shell wall thickness analysis of epoxy loaded PMMA microcapsules prepared under optimum conditions. The shell wall thickness of the prepared microcapsules was characterized by the SEM image of fracture surface of microcapsules embedded in epoxy matrix. The microcapsules were homogeneously dispersed in the epoxy matrix. For this purpose, epoxy matrix was cryogenically fractured prior to imaging. Figure 5 further confirmed the core and shell structures in the prepared microcapsules with shell wall thickness of 1.1-1.28 $\mu \mathrm{m}$ [38].

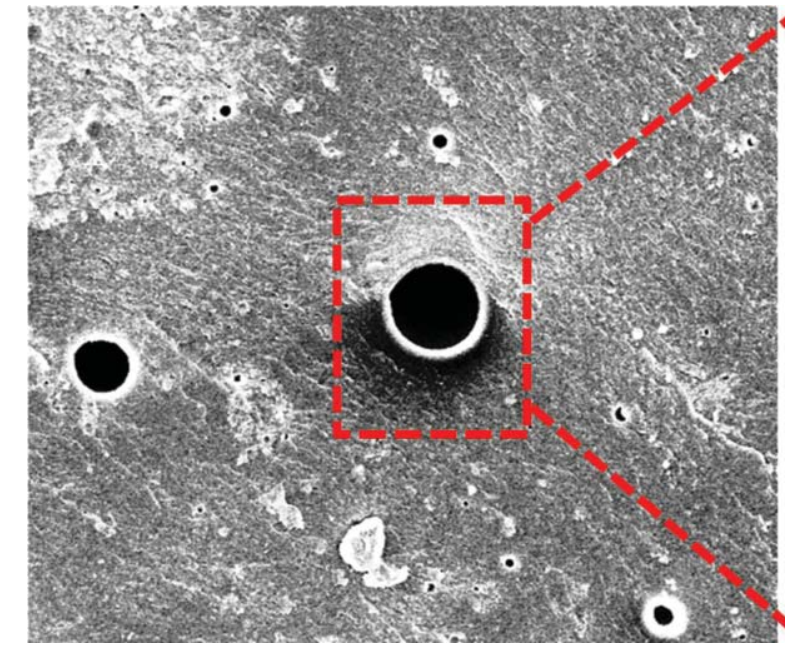

The mean particle size and particle size distribution of epoxy loaded PMMA microcapsules prepared under optimum conditions is presented in Figure 6. The average particle size of microcapsules analyzed by ImageJ software was $82 \mu \mathrm{m}$. The peak width of particle size distribution fell in the range of 1-300 $\mu \mathrm{m}$ which is quite suitable for self-healing of material. The particle size for microcapsules required for applications in the development of selfhealing polymeric composites reported in the literature lies in the range of 63 to $150 \mu \mathrm{m}$ [39].

From these studies it can be concluded that, the optimum process conditions for the preparation of microcapsules are: $10 \mathrm{wt} \%$ surfactant concentration, 3:1 core-to-shell ratio (moles), $40{ }^{\circ} \mathrm{C}$ temperature and $300 \mathrm{rpm}$ agitation speed.

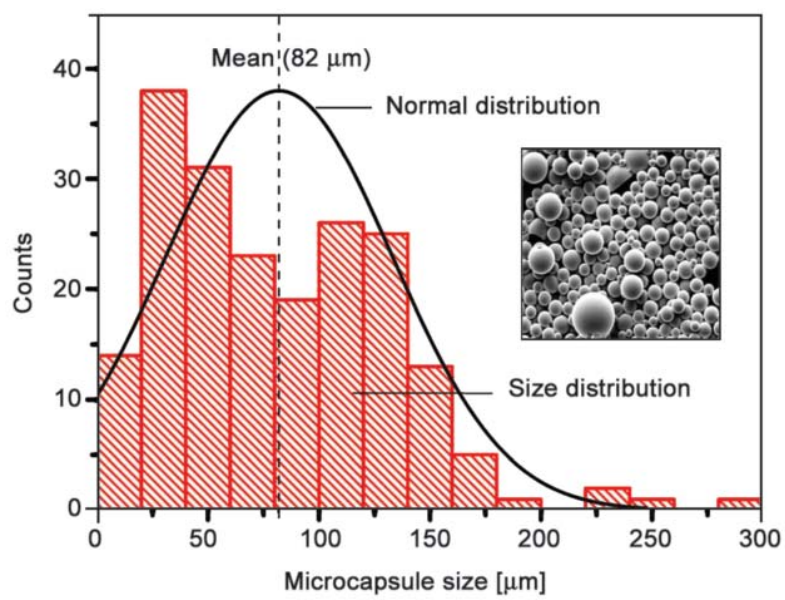

Figure 6. Particle size and particle size distribution of epoxy loaded PMMA microcapsules prepared under optimum conditions

Figure 5. Shell wall thickness analysis of microcapsules prepared under optimum conditions at 500× and $2000 \times$ magnifications 


\subsection{Contour plots}

There are four parameters used in this study such as surfactant concentration, core-to-shell ratio, temperature and agitation speed. SNR and ANOVA analysis show that in order to improve the core content of microcapsules the best contributing parameters are surfactant concentration, temperature and agitation speed. Therefore, contour plotting is done between these parameters to see how the core content relates to the two parameters at a time. The analysis of contour plots is as follows:

\subsubsection{Surfactant concentration-temperature}

Figure $7 \mathrm{a}$ indicates that how parameters surfactant concentration and temperature are related to the core content while the third parameter i.e. agitation speed

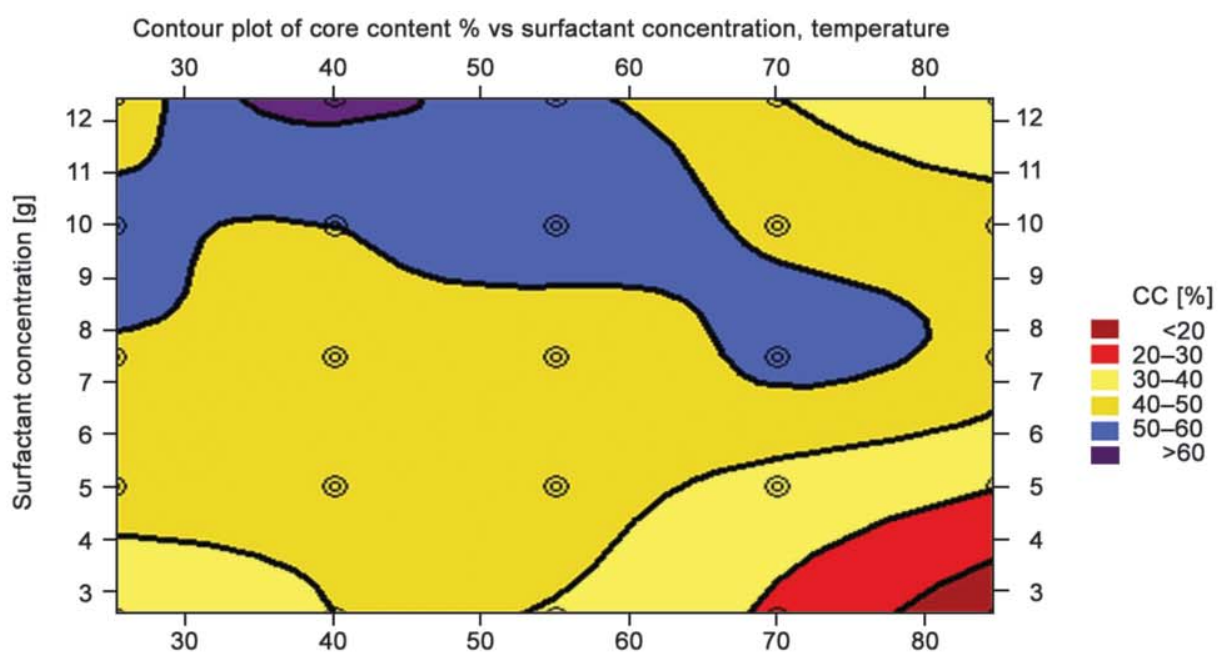

a)

Temperature $\left[{ }^{\circ} \mathrm{C}\right]$

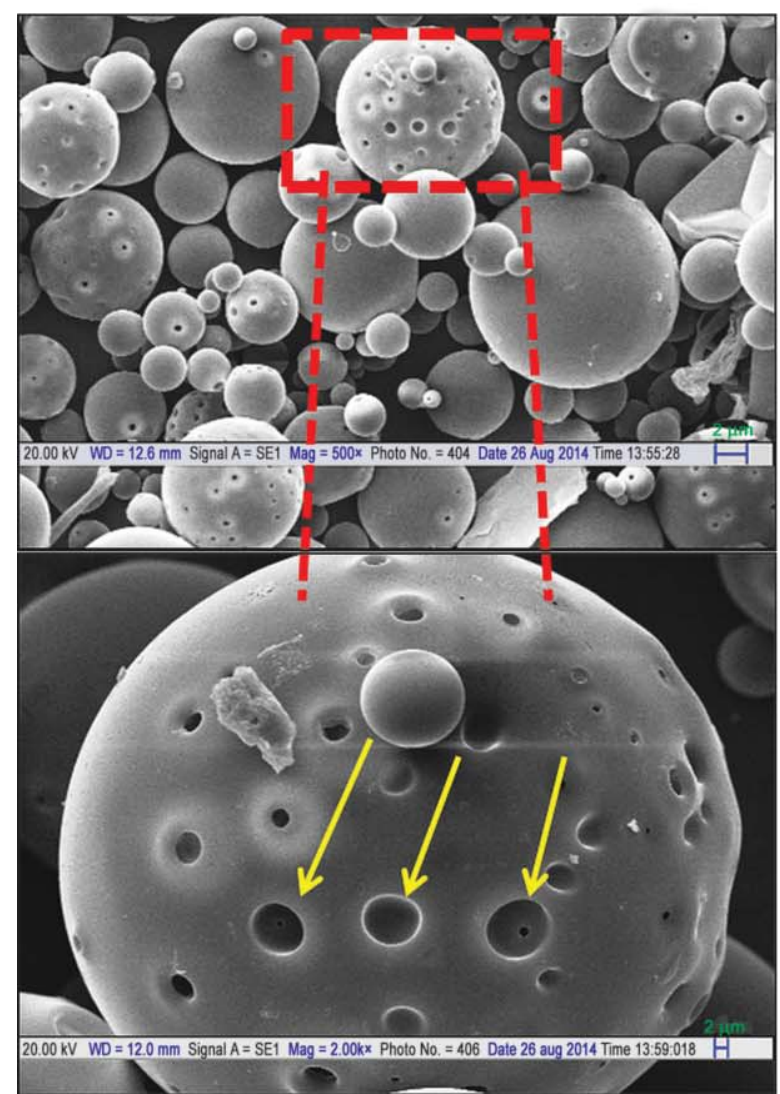

b)

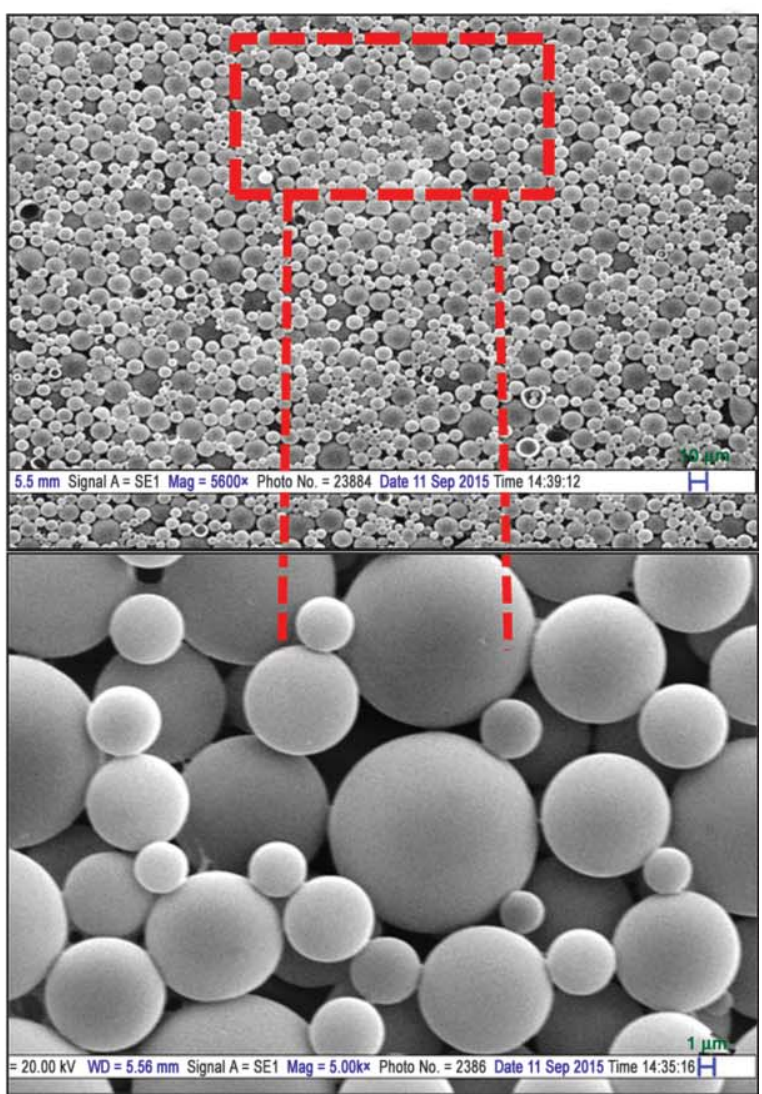

c)

Figure 7. (a) Contour plot of core content vs. surfactant concentration and temperature parameter, (b) SEM micrograph of experimental run no. 5 and its zoomed image (arrows show the presence of pores on the surface of microcapsules) and (c) SEM micrograph of experimental run no. 23 and its zoomed image 
is held constant. The analysis of plot shows that at lower level of surfactant concentration and at higher level of temperature core content can be low for example experimental run no. 5 resulted only in $11 \mathrm{wt} \%$ core content. This may be due to the fact that, under these conditions microcapsules prepared with porous surface morphology as presented in Figure $7 \mathrm{~b}$ which could be a reason for low core content. On the other hand, at higher level of surfactant concentration and at lower level of temperature core content can be high for example experimental run no. 23 resulted in $64 \mathrm{wt} \%$ core content. This may be due to the fact that under these conditions microcapsules prepared with non-porous, smooth surface morphology as presented in Figure 7c which could be a reason for high core content. Hence, the response is at its highest (greater than 60\%) at the darkest region of the plot (upper right side).

\subsubsection{Surfactant concentration-agitation speed}

Figure $8 \mathrm{a}$ indicates that how parameters surfactant concentration and agitation speed are related to the core content while the third parameter i.e. temperature is held constant. The analysis of plot shows that at lower level of surfactant concentration and at higher level of agitation speed core content can be low for example experimental run no. 4 resulted only in $28 \mathrm{wt} \%$ core content. This may be due to the fact that, under high agitation speed the shear forces would overcome the interfacial tension forces which may be resulted in the preparation of very tiny and unencapsulated microcapsules morphology as presented in Figure $8 \mathrm{~b}$ which could be a reason of low core content. This has already been reported that under lower agitation speed the microcapsules shell wall could be formed more homogeneously [40] with unbroken morphology as presented in Figure $8 \mathrm{c}$ which

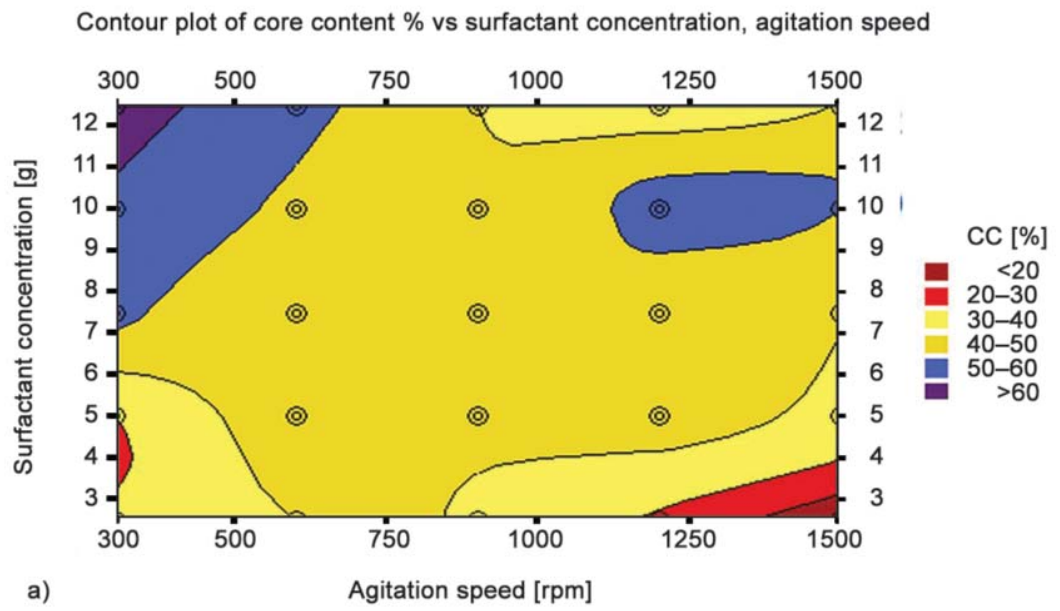

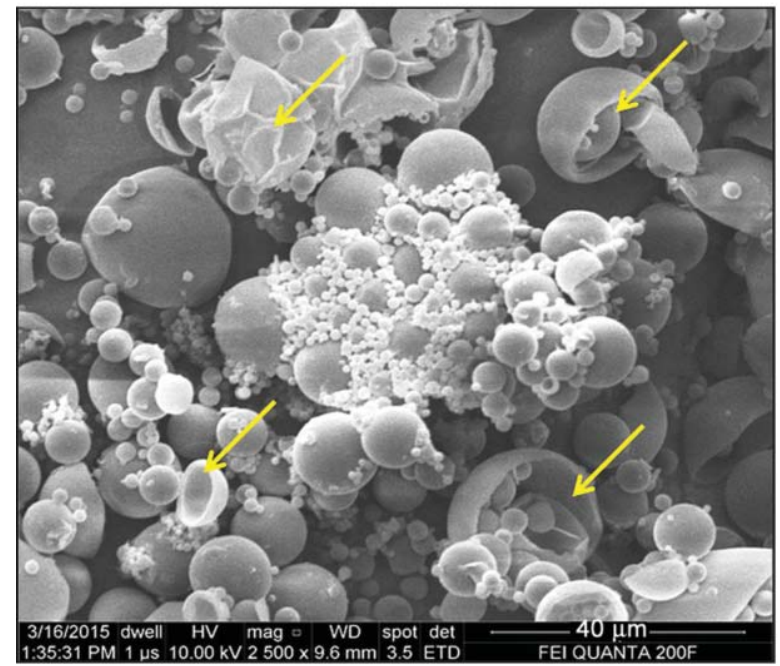

b)

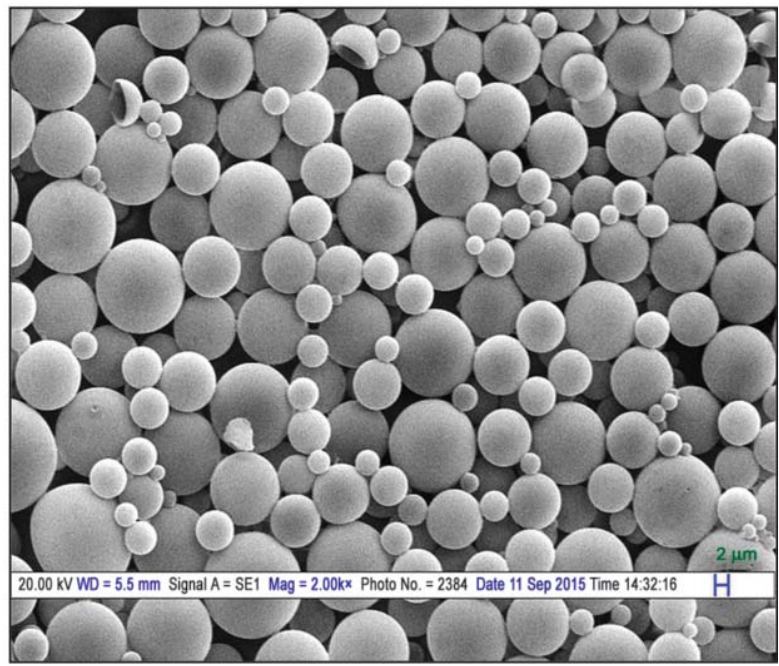

c)

Figure 8. (a) Contour plot of core content vs. surfactant concentration and agitation speed parameter, (b) SEM micrograph of experimental run no. 4 (arrows show the presence of broken morphology) and (c) SEM micrograph of experimental run no. 23 
could be a reason for high core content [for example experimental run no. 23 resulted in $64 \mathrm{wt} \%$ core content]. Hence, the response is at its highest (greater than $60 \%$ ) at the darkest region of the plot (upper right corner).

\subsubsection{Temperature-agitation speed}

Figure 9a indicates that how parameters temperature and agitation speed are related to the core content while the third parameter i.e. surfactant concentration is kept constant. The analysis of plot shows that at higher levels of temperature and agitation speed core content can be low (for example experimental run no. 21 resulted only in $32 \mathrm{wt} \%$ core content). This may be due to the fact that under these conditions microcapsules prepared with unfilled broken morphology as presented in Figure 9b which could be a reason for low core content. On the other hand, at lower levels of temperature and agitation speed core content can be high [for example experimental run no. 23 resulted in $64 \mathrm{wt} \%$ core content]. This may be due to the fact that, under these conditions microcapsules prepared with unbroken morphology as presented in Figure 9c which could be a reason for high core content. Hence, the response is at its highest (greater than 60\%) at the darkest region of the plot (lower right side).

From all contour plots analysis, it is confirmed that process parameters such as (surfactant concentration $=12.5 \mathrm{wt} \%$, core to shell ratio $=3: 1$ mole ratio, temperature $=40^{\circ} \mathrm{C}$ and agitation speed $=300 \mathrm{rpm}$ ) used in experimental run no. 23 resulted in the formation of microcapsules with high core content. From this comparative study it is concluded that, results obtained from contour plots analysis agreed well with results of Taguchi findings.

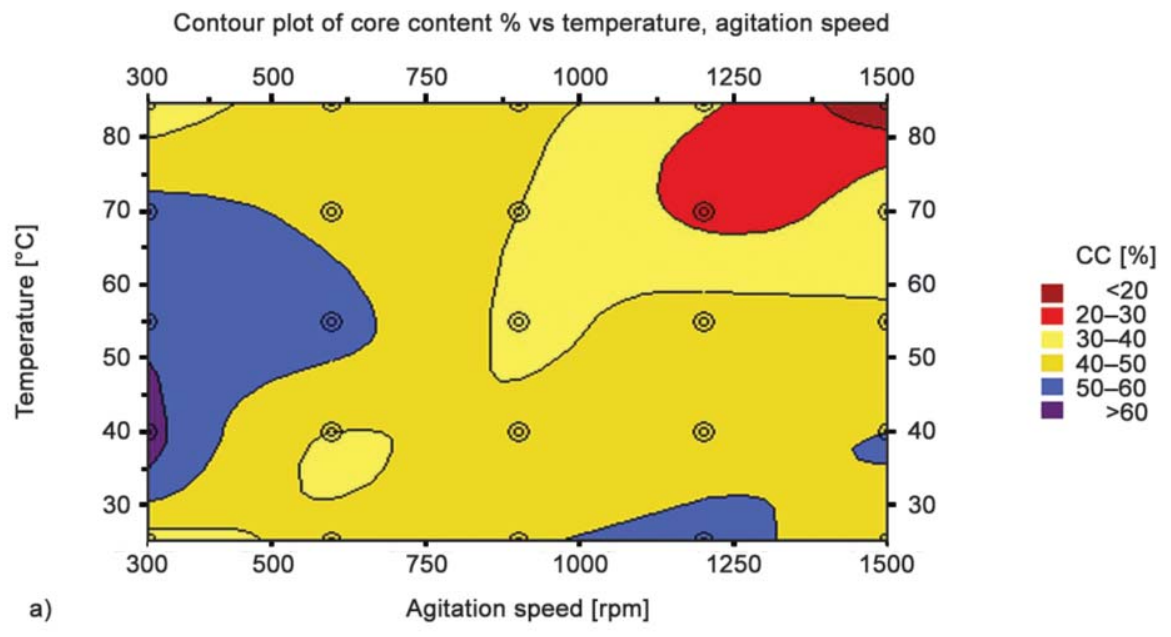

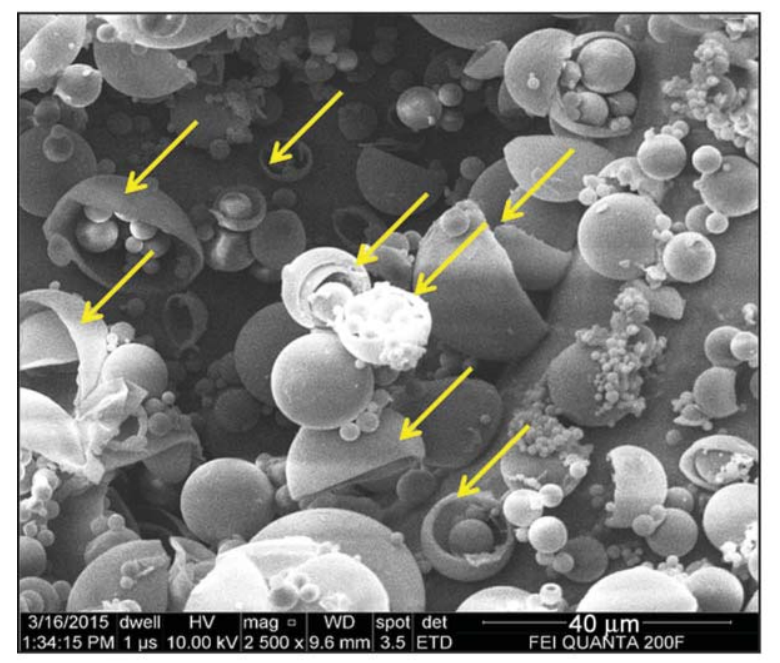

b)

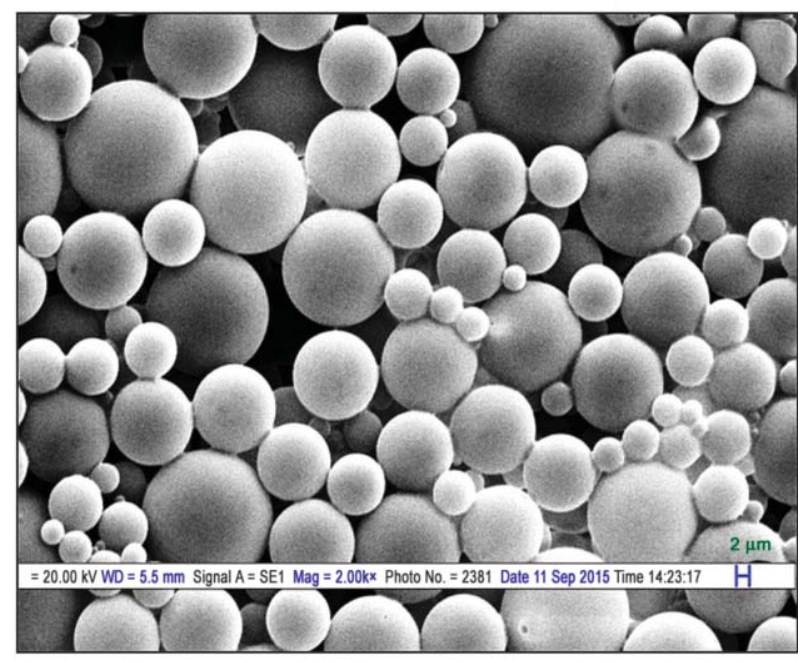

c)

Figure 9. (a) Contour plot of core content vs. temperature and agitation speed parameter, (b) SEM micrograph of experimental run no. 21 (arrows show the presence of broken morphology) and (c) SEM micrograph of experimental run no. 23 
Therefore, from this study it is concluded that the highest core content $(63.53 \%)$ could be achieved in epoxy loaded PMMA microcapsules prepared using Taguchi method optimization process parameter conditions which is much higher as compared to previously reported literatures $(54 \%)[19,21]$.

\section{Conclusions}

From this study, the following conclusions can be drawn.

- Statistically designed experiments based on Taguchi methodology were successfully performed using $\mathrm{L}_{25}$ orthogonal array to analyze the core content as a response variable.

- Predicted core content (66.36 wt\%) determined using Taguchi optimization method i.e. $10 \mathrm{wt} \%$ surfactant concentration, 3:1 core-to-shell ratio [in moles], $40^{\circ} \mathrm{C}$ temperature and $300 \mathrm{rpm}$ agitation speed agrees well with the experimental value $(63.53 \mathrm{wt} \%)$.

- ANOVA analysis confirmed that among the various parameters investigated, surfactant concentration plays a significant role in controlling the core content of PMMA microcapsules.

- The microcapsules morphology was examined using OM, SEM and TEM which confirmed that free flowing, smooth surface microcapsules can be prepared by solvent evaporation method having core/shell morphology.

- Contour plots with significant parameters agreed well with Taguchi/ANOVA analysis.

For future perspectives authors would like to acknowledge the use of hollow glass fibers as an ideal medium for storing high amount of healing components together with exploring them as structural reinforcing agents in advanced epoxy composite structures.

\section{Acknowledgements}

We acknowledge Ministry of Human Resource Development (MHRD), Government of India for providing financial assistance to one of the authors (Ms. Shilpi Sharma). Author would also like to acknowledge IIT Delhi for providing all the experimental facilities.

\section{References}

[1] Brown E. N., Kessler M. R., Sottos N. R., White S. R.: In situ poly(urea-formaldehyde) microencapsulation of dicyclopentadiene. Journal of Microencapsulation, 20, 719-730 (2003).

https://doi.org/10.1080/0265204031000154160
[2] Yuan L., Liang G-Z., Xie J-Q., Guo J., Li L.: Thermal stability of microencapsulated epoxy resins with poly (urea-formaldehyde). Polymer Degradation and Stability, 91, 2300-2306 (2006). https://doi.org/10.1016/j.polymdegradstab.2006.04.026

[3] Yuan L., Liang G., Xie J., Li L., Guo J.: Preparation and characterization of poly(urea-formaldehyde) microcapsules filled with epoxy resins. Polymer, 47, 5338-5349 (2006).

https://doi.org/10.1016/j.polymer.2006.05.051

[4] Yuan L., Gu A., Liang G.: Preparation and properties of poly (urea-formaldehyde) microcapsules filled with epoxy resins. Materials Chemistry and Physics, 110, 417-425 (2008). https://doi.org/10.1016/j.matchemphys.2008.02.035

[5] Li H., Wang R., Hu H., Liu W.: Surface modification of self-healing poly(urea-formaldehyde) microcapsules using silane-coupling agent. Applied Surface Science, 255, 1894-1900 (2008).

https://doi.org/10.1016/j.apsusc.2008.06.170

[6] Zhang T., Zhang M., Tong X-M., Feng C., Qiu J-H.: Optimal preparation and characterization of poly(ureaformaldehyde) microcapsules. Journal of Applied Polymer Science, 115, 2162-2169 (2010).

https://doi.org/10.1002/app.31329

[7] Behzadnasab M., Esfandeh M., Mirabedini S., Zohuriaan-Mehr M., Farnood R.: Preparation and characterization of linseed oil-filled urea-formaldehyde microcapsules and their effect on mechanical properties of an epoxy-based coating. Colloids and Surfaces A: Physicochemical and Engineering Aspects, 457, 16-26 (2014). https://doi.org/10.1016/j.colsurfa.2014.05.033

[8] Knop W. R., Meier M. M., Pezzin S. H.: Preparation and characterization of poly (urea-formaldehyde) microcapsules filled with dicyclopentadiene (in Portuguese). Matéria (Rio de Janeiro), 19, 266-273 (2014). https://doi.org/10.1590/S1517-70762014000300010

[9] Yuan Y., Rong M., Zhang M.: Preparation and characterization of poly(melamine-formaldehyde) walled microcapsules containing epoxy. Acta Polymerica Sinica, 5, 472-480 (2008). https://doi.org/10.3724/SP.J.1105.2008.00472

[10] Yuan L., Liang G-Z., Xie J-Q., He S-B.: Synthesis and characterization of microencapsulated dicyclopentadiene with melamine-formaldehyde resins. Colloid and Polymer Science, 285, 781-791 (2007).

https://doi.org/10.1007/s00396-006-1621-5

[11] Hu J., Chen H-Q., Zhang Z.: Mechanical properties of melamine formaldehyde microcapsules for self-healing materials. Materials Chemistry and Physics, 118, 6370 (2009).

https://doi.org/10.1016/j.matchemphys.2009.07.004

[12] Liu X., Sheng X., Lee J. K., Kessler M. R.: Synthesis and characterization of melamine-urea-formaldehyde microcapsules containing ENB-based self-healing agents. Macromolecular Materials and Engineering, 294, 389395 (2009).

https://doi.org/10.1002/mame.200900015 
[13] Tong X-M., Zhang T., Yang M-Z., Zhang Q.: Preparation and characterization of novel melamine modified poly(urea-formaldehyde) self-repairing microcapsules. Colloids and Surfaces A: Physicochemical and Engineering Aspects, 371, 91-97 (2010).

https://doi.org/10.1016/j.colsurfa.2010.09.009

[14] Schroeter J. D., Campbell J., Kimbell J. S., Conolly R. B., Clewell H. J., Andersen M. E.: Effects of endogenous formaldehyde in nasal tissues on inhaled formaldehyde dosimetry predictions in the rat, monkey, and human nasal passages. Toxicological Sciences, 138, 412 424 (2014).

https://doi.org/10.1093/toxsci/kft333

[15] Cole P., Adami H-O., Trichopoulos D., Mandel J.: Formaldehyde and lymphohematopoietic cancers: A review of two recent studies. Regulatory Toxicology and Pharmacology, 58, 161-166 (2010). https://doi.org/10.1016/j.yrtph.2010.08.013

[16] Lazenby V., Hinwood A., Callan A., Franklin P.: Formaldehyde personal exposure measurements and time weighted exposure estimates in children. Chemosphere, 88, 966-973 (2012).

https://doi.org/10.1016/j.chemosphere.2012.03.029

[17] Li Q., Siddaramaiah, Kim N. H., Hui D., Lee J. H.: Effects of dual component microcapsules of resin and curing agent on the self-healing efficiency of epoxy. Composites Part B: Engineering, 55, 79-85 (2013). https://doi.org/10.1016/j.compositesb.2013.06.006

[18] Li Q., Mishra A. K., Kim N. H., Kuila T., Lau K-T., Lee J. H.: Effects of processing conditions of poly(methylmethacrylate) encapsulated liquid curing agent on the properties of self-healing composites. Composites Part B: Engineering, 49, 6-15 (2013).

https://doi.org/10.1016/j.compositesb.2013.01.011

[19] Khan N. I., Halder S., Goyat M.: Influence of dualcomponent microcapsules on self-healing efficiency and performance of metal-epoxy composite-lap joints. The Journal of Adhesion, 2016, 1-15 (2016). https://doi.org/10.1080/00218464.2016.1193806

[20] Choudhury I., Halder S., Khan N. I., Mathur A., Nath W., Phukan A.: Effect of TETA microcapsules on self-healing ability of dual component epoxy system. Advance Material Letter, 7, 836-843 (2016).

https://doi.org/10.5185/amlett.2016.6213

[21] Ahangaran F., Navarchian A. H., Hayaty M., Esmailpour K.: Effect of mixing mode and emulsifying agents on micro/nanoencapsulation of low viscosity self-healing agents in polymethyl methacrylate shell. Smart Materials and Structures, 25, 095035/1-095035/15 (2016). https://doi.org/10.1088/0964-1726/25/9/095035

[22] Oktem H., Erzurumlu T., Uzman I.: Application of Taguchi optimization technique in determining plastic injection molding process parameters for a thin-shell part. Materials and Design, 28, 1271-1278 (2007). https://doi.org/10.1016/j.matdes.2005.12.013
[23] Kim K. D., Han D. N., Kim H. T.: Optimization of experimental conditions based on the Taguchi robust design for the formation of nano-sized silver particles by chemical reduction method. Chemical Engineering Journal, 104, 55-61 (2004).

https://doi.org/10.1016/j.cej.2004.08.003

[24] Torkaman R., Soltanieh M., Kazemian H.: Optimization of parameters for synthesis of MFI nanoparticles by Taguchi robust design. Chemical Engineering and Technology, 33, 902-910 (2010).

https://doi.org/10.1002/ceat.200900367

[25] Jahanshahi M., Sanati M., Babaei Z.: Optimization of parameters for the fabrication of gelatin nanoparticles by the Taguchi robust design method. Journal of Applied Statistics, 35, 1345-1353 (2008). https://doi.org/10.1080/02664760802382426

[26] Kim K. D., Choi D. W., Choa Y-H., Kim H. T.: Optimization of parameters for the synthesis of zinc oxide nanoparticles by Taguchi robust design method. Colloids and Surfaces A: Physicochemical and Engineering Aspects, 311, 170-173 (2007).

https://doi.org/10.1016/j.colsurfa.2007.06.017

[27] Montgomery D. C.: Introduction to statistical quality control. Wiley, New York (2009).

[28] Roy R.: A primer on the Taguchi method, competitive manufacturing series. SME, New York (1990).

[29] Taguchi G.: Introduction to quality engineering: Designing quality into products and processes. Asian Productivity Organization, Tokyo (1986).

[30] Walpole R. E., Myers R. H., Myers S. L., Ye K.: Probability and statistics for engineers and scientists. Macmillan, New York (1993).

[31] Weiss N. A., Weiss C. A.: Introductory statistics. Pearson Education, London (2012).

[32] Liu X., Zhang H., Wang J., Wang Z., Wang S.: Preparation of epoxy microcapsule based self-healing coatings and their behavior. Surface and Coatings Technology, 206, 4976-4980 (2012).

https://doi.org/10.1016/j.surfcoat.2012.05.133

[33] Sharma S., Choudhary V.: Poly(melamine-formaldehyde) microcapsules filled with epoxy resin: Effect of $\mathrm{M} / \mathrm{F}$ ratio on the shell wall stability. Materials Research Express, 4, 075307 (2017). https://doi.org/10.1088/2053-1591/aa7c8f

[34] Chandrakanth B., Kumar S. V. A., Kumar S. A., Sathish R.: Optimization and non-destructive test analysis of SS316L weldments using GTAW. Materials Research, 17, 190-195 (2014). https://doi.org/10.1590/S1516-14392013005000188

[35] Yuan Y. C., Rong M. Z., Zhang M. Q.: Preparation and characterization of microencapsulated polythiol. Polymer, 49, 2531-2541 (2008).

https://doi.org/10.1016/j.polymer.2008.03.044 
[36] Sonam, Chaudhary H., Kumar V.: Taguchi design for optimization and development of antibacterial drugloaded PLGA nanoparticles. International Journal of Biological Macromolecules, 64, 99-105 (2014). https://doi.org/10.1016/j.ijbiomac.2013.11.032

[37] Zhu D. Y., Rong M. Z., Zhang M. Q.: Self-healing polymeric materials based on microencapsulated healing agents: From design to preparation. Progress in Polymer Science, 49-50 175-220 (2015).

https://doi.org/10.1016/j.progpolymsci.2015.07.002
[38] Yi H., Deng Y., Wang C.: Pickering emulsion-based fabrication of epoxy and amine microcapsules for dual core self-healing coating. Composites Science and Technology, 133, 51-59 (2016).

https://doi.org/10.1016/j.compscitech.2016.07.022

[39] Van Benthem R. A., Ming W. M.: Self healing polymer coatings. in 'Self healing materials' (eds.: Hager M. D., van der Zwaag S., Schubert U. S.). Springer, Dordrecht, 139-159 (2007).

[40] Siddhan P., Jassal M., Agrawal A. K.: Core content and stability of n-octadecane-containing polyurea microencapsules produced by interfacial polymerization. Journal of Applied Polymer Science, 106, 786-792 (2007). https://doi.org/10.1002/app.26056 